\title{
La jurisprudence définit de manière inapplicable la notion d'urgence
}

Charles A. Steinhäuslin ${ }^{a}$, Jean-Michel Duc ${ }^{b}$

a Médecin

$b$ Avocat

\begin{abstract}
Préambule
Dans un jugement récent [1], le Tribunal fédéral a implicitement admis qu'un patient doit être transféré dans un hôpital de son canton de résidence - en l'occurrence le canton de Vaud - une fois que l'état d'urgence ayant justifié son hospitalisation dans un autre canton, ici à Genève, ait pris fin, faute de quoi une partie des frais n'est prise en charge ni par l'assurance-maladie LAMal ni par le canton de résidence.

Cette décision ne peut laisser le monde médical indifférent. Premièrement, elle a des incidences pratiques importantes pour les médecins et les établissements hospitaliers. Deuxièmement, elle n'est pas sans incidence sur le plan des coûts, notamment sur celui de la prise en charge du transfert. Troisièmement, elle risque de créer une insécurité juridique sur le plan de la responsabilité médicale. Ce sont ces quelques points que nous souhaitons examiner ci-dessous.
\end{abstract}

\section{Bref rappel de la jurisprudence}

Selon l'art. 41 al. 3 LAMal, si, pour des raisons médicales, l'assuré se soumet à un traitement hospitalier fourni par un hôpital non répertorié du canton de ré-

\section{Le patient veut une réponse médicale immédiate, sûre et concrète. Comment traiter ce phénomène}

\section{de société?}

Correspondance: Me Jean Michel Duc Avocat

Rue Etraz 12 CH-1200 Lausanne Tél. 0213314010

duc@nouvjur.ch

www.nouvjur.ch sidence, l'assureur et le canton de résidence prennent à leur charge leur part respective de rémunération au sens de l'art. 49a LAMal. A l'exception du cas d'urgence, une autorisation du canton de résidence est nécessaire. L'art. 41 al. 3bis al. 1 lettre b LAMal a précisé que sont réputés raisons médicales le cas d'urgence et le cas où les prestations nécessaires ne peuvent pas être fournies dans un hôpital répertorié du canton de résidence de l'assuré, s'il s'agit d'un traitement hospitalier.

L'enjeu de ce procès portait sur la notion d'urgence au sens des dispositions précitées et en particulier sur ses conséquences quant au droit à la prise en charge d'une hospitalisation dans un établissement se situant hors du canton de résidence de l'assuré.
Dans cette affaire, un assuré domicilié dans le canton de Vaud a été hospitalisé pendant 9 jours à Genève aux HUG pour différentes pathologies, dont 48 heures aux urgences. L'Etat de Vaud a refusé d'intervenir, considérant que le traitement était réalisable dans le canton de résidence. Le Tribunal cantonal vaudois a estimé que l'urgence était admise pour les premières 48 heures, puis, qu'elle n'était plus réalisée. Il a jugé que l'Etat de Vaud devait prendre en charge la différence du tarif en raison du séjour hospitalier extra-cantonal pour la seule hospitalisation pendant la période d'urgence, soit pour les premières 48 heures.

Le Tribunal fédéral ne remet pas directement en cause la distinction faite par le Tribunal cantonal vaudois entre la période d'urgence et la période durant laquelle l'urgence a pris fin, avec les conséquences sur la prise en charge et le remboursement des frais que cela entraîne.

\section{Notion médicale de l'urgence}

Sur le plan médical l'urgence peut être définie comme l'accueil de tout patient demandant l'intervention de soins immédiats et dont la prise en charge n'a pas été programmée. Il y a lieu ici de distinguer l'urgence médicale lourde, nécessitant de prodiguer des soins techniques spécifiques avec précocité en cas de détresse grave, de l'urgence ressentie.

Du grave polytraumatisme à la simple contusion, de l'infarctus du myocarde à la crise d'angoisse, la notion d'urgence est très diversement ressentie. Dans notre société l'on est confronté à une importante évolution de la demande de soins et sans doute à un recours croissant aux services d'urgences. C'est un phénomène qui peut probablement s'expliquer par la surmédicalisation propre à notre société; cette surmédicalisation se traduit par une surconsommation de soins. Le patient veut une réponse médicale immédiate, sûre et concrète. Comment traiter ce phénomène de société? Ce n'est pas par un accès financier restrictif aux soins en cas d'urgence lourde que l'on va juguler cette explosion de la consommation médicale. Le patient n'a aucune influence sur l'urgence médicale lourde qui ne dépend pas de sa volonté.

Dans la cause citée jugée par le Tribunal fédéral, ce n'est pas la notion d'urgence ressentie qui prévaut, mais bien celle d'urgence lourde, laquelle nécessite une intervention médicale immédiate, associée à un 
plateau technique approprié. Dans une telle situation, la prise en charge par l'assurance ne doit pas influencer le délai d'accès au traitement approprié à l'urgence et commander au sortir de celle-ci l'interruption de la chaîne du suivi thérapeutique; une telle exigence porterait atteinte à l'efficience de la prise en charge.

De même, la situation de traitement d'urgence ne doit pas être à l'origine d'une limitation à l'accès aux soins, lorsque le patient ne peut donner de consentement, médical ou économique, libre et éclairé, soit parce qu'il a perdu connaissance, soit encore parce qu'il est devenu inapte, et que l'urgence de la situation ne permet pas d'obtenir l'autorisation d'un tiers autorisé à temps pour que l'intervention soit efficace et appropriée. Toutefois, au regard de cette dernière jurisprudence susmentionnée, nous devons nous interroger sur la portée de l'absence de consentement économique du patient ou de toute personne autorisée. Qui en assumera les conséquences? S'il fallait étendre ce consentement à tel point qu'en toute situation d'urgence, le consentement économique éclairé doit être recueilli, la responsabilité civile de l'établissement serait alors engagée, ceci constituerait un handicap supplémentaire à l'accession aux soins. Le principe de précaution financière risque de commander un transfert du patient dans un autre hôpital du canton de domicile, ce qui pourrait être médicalement inapproprié. Une notion plus large de traitement médical doit prévaloir sur le traitement d'urgence immédiat. En tout état de cause, il appartient aux seuls médecins et non aux juristes de dire si l'on est en situation d'urgence et jusqu'à quel moment cette situation perdure.

\section{Notion juridique d'urgence}

Selon la jurisprudence [2], le Tribunal fédéral a rappelé qu'il y a cas d'urgence au sens de l'art. 41 al. 2 deuxième phrase LAMal, lorsque des soins médicaux doivent être administrés sans tarder et qu'il n'est pas possible ou pas approprié d'imposer à l'assuré de retourner dans son canton de résidence.

A cet égard, le canton de résidence de l'assuré est tenu de prendre en charge la différence de coûts selon l'art. 41 al. 3 LAMal lorsqu'il existe un lien de connexité matériel et temporel entre une atteinte à la santé et le caractère urgent des soins à donner. Ce lien de connexité peut même être réalisé lorsque la situation d'urgence survient à l'occasion d'un traitement administré hors du canton. Il n'est pas déterminant que la maladie nécessitant l'aide médicale urgente fût prévisible ou même qu'il existât une certaine probabilité qu'elle survînt [3]. Il suffit en principe que l'atteinte à la santé qui doit être traitée d'urgence fasse partie des risques possibles de l'intervention médicale volontaire qui est effectuée hors du canton de résidence. Constituent de tels risques toutes les maladies qui peuvent être favorisées par le traitement volontaire administré hors du canton de résidence. Il n'est

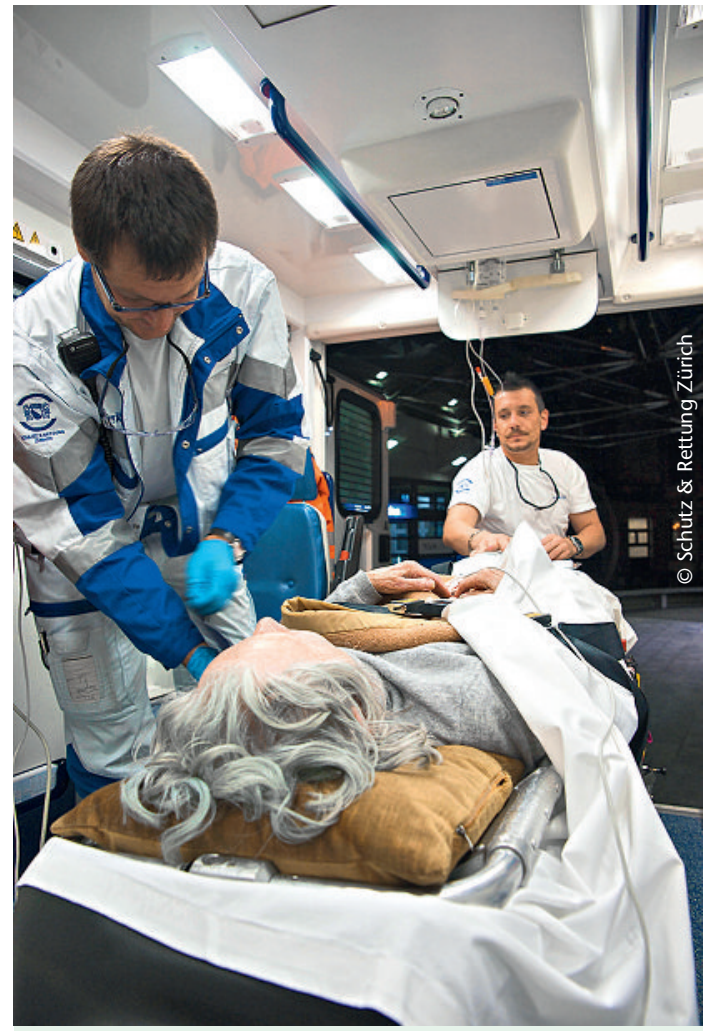

Le principe de précaution financière risque de commander un transfert du patient dans un autre hôpital du canton de domicile, ce qui pourrait être médicalement inapproprié.

pas déterminant à cet égard qu'il existe probablement, ou même au degré de la vraisemblance prépondérante, un lien de causalité naturelle entre l'atteinte à la santé qui commande des soins d'urgence et le traitement préalable (hors du canton de résidence) ou que cette atteinte soit considérée comme une maladie indépendante du point de vue thérapeutique et diagnostique. Il en va différemment seulement s'il apparaît, au degré de la vraisemblance prépondérante, que la maladie nécessitant des soins urgents serait également survenue sans le traitement volontaire hors du canton [4].

\section{Conséquence juridique}

Le fait de limiter le droit aux pleines prestations LAMal aux seules heures d'hospitalisation en urgence et d'exiger ensuite le transfert vers un hôpital du canton de résidence ne paraît guère compatible avec la sécurité du patient, tout transfert étant source de risques supplémentaires. Par ailleurs, la pratique médicale et hospitalière s'en trouverait inutilement compliquée par les démarches administratives induites par le transfert au sortir des urgences vers un autre hôpital.

Enfin, un défaut d'information au patient ou à sa famille et un séjour prolongé dans l'hôpital hors canton pourraient entraîner la responsabilité du médecin ou de l'hôpital sur le plan civil au titre de la 
violation du consentement économique éclairé du patient. Ces derniers pourraient alors devoir assumer la différence tarifaire. D'autre part, un déplacement au sortir des urgences ne peut qu'accroître les risques pour le patient. Que l'on songe à ceux en rapport avec son déplacement, avec le transfert des données médicales et avec le changement de l'équipe soignante. Enfin, qui prendrait à sa charge les coûts induits par ce transfert? Certainement pas le patient ni l'assurance LAMal. Serait-ce à charge de l'établissement hors canton du domicile?

\section{Propositions}

A notre avis, la prise en charge en cas d'urgence au sens de l'art. 41 al. 3 LAMal ne devrait pas se limiter aux seules heures d'hospitalisation en urgence; une telle limitation n'est guère compatible avec la médecine hospitalière. Elle ne répond pas non plus au critère d'économicité, puisqu'en définitive le coût d'hospitalisation est augmenté par celui du transfert, lequel comprend non seulement le déplacement du patient proprement dit, soit par exemple les frais d'ambulance, mais encore tous les frais induits par le transfert, soit la transmission et la prise de connaissance du dossier, les nouveaux examens médicaux nécessités par le changement d'équipe de soins, etc.

A notre avis, des motifs relevant de la sécurité médicale et économique justifieraient que l'on renonce au transfert hospitalier d'un patient sortant des soins intensifs. Une fois admise l'hospitalisation en urgence dans un établissement hospitalier hors canton du domicile, celle-ci devrait pouvoir se poursuivre pendant toute la phase d'hospitalisation en soins aigus au sens de l'art. 39 LAMal.

\section{«La solution résiderait dans un transfert des compétences des cantons à la Confédération, avec une planification hospitalière fédérale.»}

\section{Conclusions}

Il est vraisemblable que cette jurisprudence restrictive de la notion d'urgence s'inscrive dans le cadre des intentions politiques de limiter les coûts de la santé. Or, en réalité, elle ne conduit à aucune économie. Bien au contraire, elle génère des dépenses supplémentaires en rapport avec le transfert et fait courir des risques supplémentaires inutiles au patient causés par celuici.

Pour que le traitement puisse demeurer véritablement efficace, approprié et répondre au critère d'économicité, il faut se garder de ce type de décisions. Notre système de santé doit repenser la prise en charge de la médecine hospitalière. Pour d'aucuns, la solution résiderait dans un transfert des compétences des cantons à la Confédération, avec une planification hospitalière fédérale. Pour d'autres, elle est ailleurs et relève plutôt de la responsabilité individuelle; elle est le prix à payer pour les nouvelles mesures d'investigation et de traitement qui permettent de lutter contre la maladie et fait reculer la mort. Il s'agit assurément d'un choix de société; il y a lieu de savoir quel niveau de santé nous voulons en Suisse.

Cela étant, la disposition de l'art. 41 al. 3 LAMal est obsolète; son application stricte conduit à un résultat contraire au but de la loi, puisque, pour des seuls motifs financiers, elle entraînera un transfert de patients d'un hôpital à un autre en générant des coûts et des risques médicaux supplémentaires pour les patients. Ajoutons encore que la nouvelle tarification SwissDRG ne change rien à la problématique posée par cette jurisprudence, bien au contraire, elle ne fait que confirmer l'absence d'économicité de la jurisprudence que nous critiquons. En effet, si à compter du $1^{\text {er }}$ janvier 2012, les traitements stationnaires dans les hôpitaux devront être facturés en règle générale au moyen d'une structure de forfaits par cas uniforme s'appliquant dans toute la Suisse, le système de la participation cantonale demeure et se réfère expressément à la notion d'urgence. Ainsi, la nouvelle teneur de l'art. 41 al. 3 LAMal prévoit que «Si, pour des raisons médicales, soit en particulier en cas d'urgence, l'assuré se soumet à un traitement hospitalier fourni par un hôpital non répertorié du canton de résidence, l'assureur et le canton de résidence prennent à leur charge leur part respective de rémunération au sens de l'art. 49a. A l'exception du cas d'urgence, une autorisation du canton de résidence est nécessaire.»

\footnotetext{
Références

1 Arrêt du 11 octobre 2010 (9C_151/2010).

2 Arrêt du 31 mars 2009 (9C 812/2008), ATFA K 128/01 in RAMA $2002 \mathrm{n}^{\circ} \mathrm{KV} 231$ p. 475.

3 ATFA K 81/05 in RAMA $2006 n^{\circ}$ KV 369 p. 232.

4 ATFA K 117/06.
} 